\title{
Generalized Schwarzian derivatives for generalized fractional linear transformations
}

\author{
by John RYAN (Sydney)
}

\begin{abstract}
Generalizations of the classical Schwarzian derivative of complex analysis have been proposed by Osgood and Stowe [12, 13], Carne [5], and Ahlfors [3]. We present another generalization of the Schwarzian derivative over vector spaces.
\end{abstract}

Introduction. Our approach is to define an analogue of the Schwarzian derivatives in $\mathbb{R} \cup\{\infty\}$ using the Clifford algebra generated from $\mathbb{R}^{n}$. More precisely, we use Vahlen's group of Clifford matrices to construct a "derivative" which in appearance bears an extremely close resemblance to the classical Schwarzian derivative. As conformal transformations in dimensions greater than two correspond to Möbius transformations we are forced to introduce a family of Schwarzians in higher dimensions. We show that a $C^{3}$ diffeomorphism annihilated by this family of Schwarzian derivatives is, up to a linear isomorphism, a Möbius transformation. We also show that these generalized Schwarzian derivatives possess a conformal invariance under Möbius transformations, and contain the generalized Schwarzian derivatives described by Ahlfors [3]. Unfortunately, this work also tells us that the method used for obtaining the chain rule for the classical Schwarzian derivative (see [10]) breaks down in higher dimensions.

Motivated by the fact that the analogue of Vahlen's group of Clifford matrices over Minkowski space is $U(2,2)$ we show that the fractional linear transformations associated with $U(2,2), \operatorname{Sp}(n, \mathbb{R})$, the real symplectic group, and $H(n, n)$, the quaternionic unitary group, all have Schwarzian derivatives associated with them. These transformations have previously been described in $[7,9]$, and elsewhere. We also show that the conformal group over $\mathbb{R}^{p, q}$ has a generalized Schwarzian derivative.

Preliminaries. From $\mathbb{R}^{n}$ we may construct a Clifford algebra $A_{n}$. This can be done $[4,14]$ by taking an orthonormal basis $\left\{e_{j}\right\}_{j=1}^{n}$ of $\mathbb{R}^{n}$ and 
introducing the basis

$$
1, e_{1}, \ldots, e_{n}, \ldots, e_{j_{1}} \ldots e_{j_{r}}, \ldots, e_{1} \ldots e_{n}
$$

of $A_{n}$, where 1 is the identity and $j_{1}<\ldots<j_{r}$ with $1 \leq r \leq n$. Moreover, the elements $e_{1}, \ldots, e_{n}$ satisfy the identity

$$
e_{i} e_{j}+e_{j} e_{i}=-2 \delta_{i j} 1
$$

within $A_{n}$, where $\delta_{i j}$ is the Kronecker delta. We now have $\mathbb{R}^{n} \subseteq A_{n}$ and each non-zero vector $x \in \mathbb{R}^{n} \backslash\{0\}$ has a multiplicative inverse $x^{-1}=-x /|x|^{2} \in$ $\mathbb{R}^{n}$, which corresponds to the Kelvin inverse of a vector.

Writing $x$ as $x_{1} e_{1}+\ldots+x_{n} e_{n}$ we may obtain

$$
e_{1}\left(x_{1} e_{1}+\ldots+x_{n} e_{n}\right) e_{1}=-x_{1} e_{1}+x_{2} e_{2}+\ldots+x_{n} e_{n},
$$

which describes a reflection along the line spanned by $e_{1}$. In greater generality, for each $y \in S^{n-1}$ the element $y x y$ is a vector, and this action describes a reflection along the line spanned by $y$. By induction, for $y_{1}, \ldots, y_{k} \in S^{n-1}$ the element $y_{1} \ldots y_{k} x y_{k} \ldots y_{1}$ is a vector and this action describes an orthogonal transformation of $\mathbb{R}^{n}$. The element $y_{1} \ldots y_{k}$ is an element lying in $A_{n}$. This group is called $\operatorname{Pin}(n)$ (see [4]). More formally, we have

$\operatorname{Pin}(n)=\left\{a \in A_{n}: a=y_{1} \ldots y_{k}\right.$ where $k \in \mathbb{N}$ and $y_{j} \in S^{n-1}$ for $\left.1 \leq j \leq k\right\}$.

In [4] it is shown that $\operatorname{Pin}(n)$ is a double covering of $O(n)$, the orthogonal group (i.e. there is a surjective group homomorphism $\Theta: \operatorname{Pin}(n) \rightarrow O(n)$ such that $\operatorname{ker} \Theta \cong \mathbb{Z}_{2}$ ).

We also need the antiautomorphism $\sim: A_{n} \rightarrow A_{n}, e_{j_{1}} \ldots e_{j_{r}} \mapsto e_{j_{r}} \ldots e_{j_{1}}$. It is usual to write $\widetilde{X}$ for $\sim(X)$, where $X \in A_{n}$ (see [14]). If $a=y_{1} \ldots y_{k} \in$ $\operatorname{Pin}(n)$ then $y_{k} \ldots y_{1}=\widetilde{a}$.

Besides $\sim$ we need the antiautomorphism $-: A_{n} \rightarrow A_{n}, e_{j_{1}} \ldots e_{j_{r}} \mapsto$ $(-1)^{r} e_{j_{r}} \ldots e_{j_{1}}$. Again, it is usual [14] to write $\bar{X}$ for $-(X)$. If we write $X$ as $x_{0}+\ldots+x_{1 \ldots n} e_{1} \ldots e_{n}$ then we can easily deduce that the identity part of $X \bar{X}$ is $x_{0}^{2}+\ldots+x_{1 \ldots n}^{2}$. So $A_{n}$ is a trace algebra.

Following Vahlen [15] and Mass [11], Ahlfors [1, 2] has used Clifford algebras to describe properties of Möbius transformations in $\mathbb{R}^{n} \cup\{\infty\}$.

We shall now briefly redescribe these transformations.

The transformations

(a) $T: \mathbb{R}^{n} \cup\{\infty\} \rightarrow \mathbb{R}^{n} \cup\{\infty\}, T: \mathbb{R}^{n} \rightarrow \mathbb{R}^{n}$ is an orthogonal transformation and $T(\infty)=\infty$,

(b) $R: \mathbb{R}^{n} \cup\{\infty\} \rightarrow \mathbb{R}^{n} \cup\{\infty\}, x \mapsto x+v$ for $x \in \mathbb{R}^{n}$ and $v \in \mathbb{R}^{n}$,

$$
\infty \mapsto \infty,
$$


(c) $D: \mathbb{R}^{n} \cup\{\infty\} \rightarrow \mathbb{R}^{n} \cup\{\infty\}, x \mapsto \lambda x$ for $x \in \mathbb{R}^{n}$ and $\lambda \in \mathbb{R}$, $\infty \mapsto \infty$,

(d) In : $\mathbb{R}^{n} \cup\{\infty\} \rightarrow \mathbb{R}^{n} \cup\{\infty\}, x \mapsto x^{-1}$ for $x \in \mathbb{R}^{n} \backslash\{0\}$,

$$
\begin{aligned}
\infty & \mapsto 0, \\
0 & \mapsto \infty,
\end{aligned}
$$

are all special examples of Möbius transformations.

DEFINITION 1. The group of diffeomorphisms of $\mathbb{R}^{n} \cup\{\infty\}$ generated by the transformations (a)-(d) is called the Möbius group, and is denoted by $\operatorname{Möb}(n)$. An element of $\operatorname{Möb}(n)$ is called a Möbius transformation.

When $n=1$ the Clifford algebra is the complex field, and in this case it is extremely well known that a sense preserving Möbius transformation in two real dimensions can be written as $(a z+b)(c z+d)^{-1}$ where $\left(\begin{array}{ll}a & b \\ c & d\end{array}\right) \in \operatorname{SL}(2, \mathbb{C})$ and $z \in \mathbb{C} \cup\{\infty\}$.

In higher dimensions we have:

Definition 2. A matrix $\left(\begin{array}{ll}a & b \\ c & d\end{array}\right)$ with $a, b, c, d \in A_{n}$ and

(i) $a=a_{1} \ldots a_{n_{1}}, b=b_{1} \ldots b_{n_{2}}, c=c_{1} \ldots c_{n_{3}}, d=d_{1} \ldots d_{n_{4}}$, with $n_{1}, n_{2}, n_{3}, n_{4} \in \mathbb{N}$ and $a_{i}, b_{j}, c_{k}, d_{l} \in \mathbb{R}^{n}$ for $1 \leq i \leq n_{1}, 1 \leq j \leq n_{2}$, $1 \leq k \leq n_{3}, 1 \leq l \leq n_{4}$

(ii) $a \widetilde{c}, \widetilde{c} d, d \widetilde{b}, \widetilde{b} a \in \mathbb{R}^{n}$,

(iii) $a \widetilde{d}-b \widetilde{c} \in \mathbb{R} \backslash\{0\}$,

is called a Vahlen matrix.

From (2) and (i) we see that if $a \widetilde{c}$ is in $\mathbb{R}^{n}$ then so is $\widetilde{c}(a \widetilde{c}) c=\widetilde{c} a(\widetilde{c} c)$. But $\widetilde{c} c \in \mathbb{R}$, and so $\widetilde{c} a \in \mathbb{R}^{n}$, Consequently, (ii) is equivalent to saying $\widetilde{c} a, d \widetilde{c}, \widetilde{b} d, a \widetilde{b} \in \mathbb{R}^{n}$.

As $\widetilde{c} d \in \mathbb{R}^{n}$ we have $\widetilde{c} c x+\widetilde{c} d \in \mathbb{R}^{n}$ for each $x \in \mathbb{R}^{n}$, so if $c \neq 0$ then $c x+d$ is invertible in $A_{n}$ for all but one value of $x \in \mathbb{R}^{n} \cup\{0\}$. If $c=0$ then it follows from Definition 2 that $d$ is invertible in $A_{n}$. Consequently, $(a x+b)(c x+d)^{-1}$ is a well defined element of $A_{n}$ for all but one value of $x \in \mathbb{R}^{n} \cup\{0\}$.

When $c \neq 0$ we have

$$
(a x+b)(c x+d)^{-1}=a c^{-1}+\lambda(c x \widetilde{c}+d \widetilde{c})^{-1}
$$

where $\lambda \in \mathbb{R} \backslash\{0\}$, and when $c=0$,

$$
(a x+b)(c x+d)^{-1}=a x d^{-1}+b d^{-1} .
$$

Both (3) and (4) are Möbius transformations.

From (3) and (4) we have 
LEMMA 1 [1]. Each Vahlen matrix can be expressed as a finite product of the special Vahlen matrices

$$
\left(\begin{array}{cc}
a & 0 \\
0 & \widetilde{a}^{-1}
\end{array}\right), \quad\left(\begin{array}{cc}
\lambda^{1 / 2} & 0 \\
0 & \lambda^{-1 / 2}
\end{array}\right), \quad\left(\begin{array}{ll}
1 & v \\
0 & 1
\end{array}\right), \quad\left(\begin{array}{ll}
0 & 1 \\
1 & 0
\end{array}\right)
$$

where $a \in \operatorname{Pin}(n), \lambda \in \mathbb{R}^{+}$, and $v \in \mathbb{R}^{n}$.

These special Vahlen matrices transform into special Möbius transformations (a)-(d). Using this fact, the identities (3) and (4), and Lemma 1 it is straightforward to deduce

Proposition 1 [1]. The set $V(n)$ of Vahlen matrices over $\mathbb{R}^{n}$ forms a group under matrix multiplication, and the projection

$$
p: V(n) \rightarrow \operatorname{Möb}(n), \quad\left(\begin{array}{ll}
a & b \\
c & d
\end{array}\right) \mapsto(a x+b)(c x+d)^{-1},
$$

is a surjective group homomorphism.

By trying to determine the Vahlen matrices for which the equation

$$
x=(a x+b)(c x+d)^{-1}
$$

holds for all $x \in \mathbb{R}^{n}$ we may use (3) and (4) to obtain

Proposition 2.

$$
\operatorname{Ker}(p)=\left\{\left(\begin{array}{cc}
\lambda & 0 \\
0 & \lambda
\end{array}\right),\left(\begin{array}{cc}
\lambda e_{1} \ldots e_{n} & 0 \\
0 & -\lambda\left(e_{1} \ldots e_{n}\right)^{-1}
\end{array}\right): \lambda \in \mathbb{R} \backslash\{0\}\right\} .
$$

Consequently, the group $V(n) \backslash \mathbb{R}^{+}$is a four-fold covering group of $\operatorname{Möb}(n)$. Now,

$$
V(n) \backslash \mathbb{R}^{+} \cong\left\{\left(\begin{array}{ll}
a & b \\
c & d
\end{array}\right) \in V(n): a \widetilde{d}-b \widetilde{c}= \pm 1\right\} .
$$

The subgroup

$$
V_{+}(n)=\left\{\left(\begin{array}{ll}
a & b \\
c & d
\end{array}\right) \in V(n): a \widetilde{d}-b \widetilde{c}=1\right\}
$$

of $V(n) \backslash \mathbb{R}^{+}$is a natural generalization of $\mathrm{SL}(2, \mathbb{R})$.

The Vahlen matrices introduced here are not quite the same as those described in [1]. We now introduce those matrices:

Definition 3. A matrix $\left(\begin{array}{ll}a & b \\ c & d\end{array}\right)$ with $a, b, c, d \in A_{n}$ and

(i) $a=a_{1} \ldots a_{n_{1}}, b=b_{1} \ldots b_{n_{2}}, c=c_{1} \ldots c_{n_{3}}, d=d_{1} \ldots d_{n_{4}}$, with $a_{i}, b_{j}, c_{k}, d_{l} \in \mathbb{R}+\mathbb{R}^{n}$,

(ii) $\bar{a} c, \bar{c} d, \bar{d} b, \bar{b} a \in \mathbb{R}+\mathbb{R}^{n}$,

(iii) $a \tilde{d}-b \widetilde{c} \in \mathbb{R} \backslash\{0\}$,

where $\mathbb{R}+\mathbb{R}^{n}$ is spanned by $1, e_{1}, \ldots, e_{n}$, is called a refined Vahlen matrix. 
We denote the set of refined Vahlen matrices over $\mathbb{R}+\mathbb{R}^{n}$ by $V_{0}(n)$. By similar arguments to those given above we find [1] that $V_{0}(n)$ is a group. The subgroup

$$
V_{0,+}(n)=\left\{\left(\begin{array}{ll}
a & b \\
c & d
\end{array}\right) \in V_{0}(n): a \widetilde{d}-b \widetilde{c}=1\right\}
$$

is a generalization of $\mathrm{SL}(2, \mathbb{C})$. Indeed, $V_{0,+}(1)=\mathrm{SL}(2, \mathbb{C})$.

Other properties of these types of matrices can be found in $[6]$.

1. Now suppose that $A$ is a real normed algebra with an identity, and $U(A)$ is the open set of invertible elements in $A$. Suppose that $V$ is a domain in $\mathbb{R}^{n}$ and $f: V \rightarrow U(A)$ is a $C^{1}$ function. For $y \in S^{n-1}$ we shall let $f(x)_{y}$ denote the partial derivative of $f$ at $x$ in the direction of $y$.

The following simple result is crucial to all that follows:

Proposition 3. Suppose that $f(x)^{-1}$ denotes the algebraic inverse of $f(x)$. Then $\left(f(x)^{-1}\right)_{y}=-f(x)^{-1} f(x)_{y} f(x)^{-1}$.

Proof.

$$
\begin{aligned}
\frac{1}{h}\left(f(x+h y)^{-1}-f(x)^{-1}\right) & =\frac{1}{h} f(x+h y)^{-1}(f(x)-f(x+h y)) f(x)^{-1} \\
& =-f(x+h y)^{-1}\left(\frac{f(x+h y)-f(x)}{h}\right) f(x)^{-1} .
\end{aligned}
$$

So

$$
\lim _{h \rightarrow 0} \frac{1}{h}\left(f(x+h y)^{-1}-f(x)^{-1}\right)=-f(x)^{-1} f(x)_{y} f(x)^{-1} .
$$

This result is an elementary generalization of the basic result that for $f: \mathbb{R} \backslash\{0\} \rightarrow \mathbb{R} \backslash\{0\}, f(x)=1 / x$, we have $(d f / d x)(x)=-1 / x^{2}$.

2. From Proposition 3 and (3) and (4) we have

Lemma 2. Suppose that $\left(\begin{array}{ll}a & b \\ c & d\end{array}\right) \in V(n) \backslash \mathbb{R}^{+}$and $\Phi(z)=(a z+b)(c x+d)^{-1}$. Then for each $y \in S^{n-1}$ we have

$$
\Phi(x)_{y}= \begin{cases}-\lambda \widetilde{c}^{-1}\left(x+c^{-1} d\right)^{-} y\left(x+c^{-1} d\right)^{-1} c^{-1} & \text { if } c \neq 0 \\ a y d^{-1} & \text { otherwise } .\end{cases}
$$

From Lemma 2 and Proposition 3 it is now easy to deduce the following formula:

$$
\Phi(x)_{y y y} \Phi(x)_{y}^{-1}-\frac{3}{2}\left\{\Phi(x)_{y y} \Phi(x)_{y}^{-1}\right\}^{2}=0 .
$$

Here $\Phi(x)_{y y y}$ and $\Phi(x)_{y y}$ mean respectively the third and second partial derivatives of $\Phi$ at $x$ in the direction of $y$. Moreover, $\Phi(x)_{y}^{-1}$ denotes the Kelvin inverse of the vector $\Phi(x)_{y}$. (From the expressions appearing in Lemma 2 it is straightforward to see that $\Phi(x)_{y}$ is a non-zero vector.) 
Expression (5) is very similar in appearance to the classical Schwarzian derivative of a Möbius transformation in $\mathbb{C} \cup\{\infty\}$ (see for example [10]).

Lemma 3. Suppose that $w: V \hookrightarrow \mathbb{R}^{n}$ is a $C^{1}$ diffeomorphism. Then $w(x)_{y}$ is a non-zero vector for each $x \in V$.

Using Lemma 3 we can now make the following definition:

Definition 4. Suppose that $w: V \hookrightarrow \mathbb{R}^{n}$ is a $C^{3}$ diffeomorphism. Then we define $\{S, w\}_{y}$ to be $w_{y y y} w_{y}^{-1}-\frac{3}{2}\left(w_{y y} w_{y}^{-1}\right)^{2}$, and we call $\{S, w\}_{y}$ the Schwarzian derivative of $w$ in the direction of $y \in S^{n-1}$.

$\{S, w\}_{y}$ takes its values in the Lie subalgebra of $A_{n}$ spanned by $\left\{1, e_{i} e_{j}\right.$, $\left.e_{i} e_{j} e_{k} e_{l}: 1 \leq i<k<l \leq n\right\}$.

From Proposition 3 we have

Lemma 4. Suppose that $w: V \hookrightarrow \mathbb{R}^{n}$ is a $C^{3}$ diffeomorphism. Then

$$
\left(w(x)_{y y} w(x)_{y}^{-1}\right)_{y}=w(x)_{y y y} w(x)_{y}^{-1}-\left(w_{y y}(x) w(x)_{y}^{-1}\right)^{2},
$$

where $\left(w(x)_{y y} w(x)_{y}^{-1}\right)_{y}$ denotes the partial derivative of $w(x)_{y y} w(x)_{y}^{-1}$ at $x$ in the direction of $y$.

As a consequence of Lemma 4 we have Then

Proposition 4. Suppose that $w: V \hookrightarrow \mathbb{R}^{n}$ is a $C^{3}$ diffeomorphism.

$$
\{S, w\}_{y}=\left(w_{y y} w_{y}^{-1}\right)_{y}-\frac{1}{2}\left(w_{y y} w_{y}^{-1}\right)^{2} .
$$

Expression (6) is completely analogous to the other well known form of the classical Schwarzian (see [10]).

We shall now try to determine solutions to the equation

$$
\{S, w\}_{y}=0 .
$$

First we note

Lemma 5. Suppose that $L: \mathbb{R}^{n} \rightarrow \mathbb{R}^{n}$ is an isomorphism. Then $\{S, L\}_{y}$ $=0$ for all $y \in S^{n-1}$.

The fact that $L$ is a solution to our generalized Schwarzian represents a departure from the results in complex analysis, and is a consequence of the fact that the Schwarzian presented here is dependent on our choice of $y$.

Bearing this in mind we are led to the following result:

Proposition 5. Suppose that $w: V \hookrightarrow \mathbb{R}^{n}$ is a $C^{3}$ diffeomorphism and $\{S, w\}_{e_{1}}=0$. Suppose also that $w_{e_{1} e_{1}} \neq 0$. Then there exist $C^{3}$ maps $a\left(x_{2}, \ldots, x_{n}\right), b\left(x_{2}, \ldots, x_{n}\right), c\left(x_{2}, \ldots, x_{n}\right)$ and $d\left(x_{2}, \ldots, x_{n}\right)$ such that

$$
w(x)=\left(a\left(x_{2}, \ldots, x_{n}\right)+x_{1}\right)^{-1} b\left(x_{2}, \ldots, x_{n}\right)+c\left(x_{2}, \ldots, x_{n}\right) .
$$


Proof. First we set $w(x)_{e_{1} e_{1}} w(x)_{e_{1}}^{-1}=v(x)$. So the equation $\{S, w\}_{e_{1}}$ $=0$ becomes

$$
\frac{\partial v}{\partial x_{1}}=\frac{1}{2} v^{2}
$$

As $w(x)_{e_{1} e_{1}} \neq 0$ we find that $v$ is invertible in the Clifford algebra. So (8) is equivalent to

or

$$
v^{-1} \frac{\partial v}{\partial x_{1}} v^{-1}=\frac{1}{2}
$$

But from Proposition 3 we have

$$
-v^{-1} \frac{\partial v}{\partial z_{1}} v^{-1}=\frac{1}{2}
$$

$$
v^{-1} \frac{\partial v}{\partial x_{1}} v^{-1}=\frac{\partial}{\partial x_{1}}\left(v^{-1}\right) .
$$

So $\left(\partial / \partial x_{1}\right)\left(v^{-1}\right)=-1 / 2$. Consequently,

$$
v(x)^{-1}=-\frac{1}{2}\left(x_{1}+a\left(x_{2}, \ldots, x_{n}\right)\right) .
$$

As $v(x)$ is invertible in $A_{n}, x_{1}+a\left(x_{2}, \ldots, x_{n}\right)$ must be invertible in $A_{n}$. So

$$
-2\left(x_{1}+a\left(x_{2}, \ldots, x_{n}\right)\right)^{-1}=v(x) .
$$

We now set $\partial w / \partial x_{1}=u(x)$. So we have

$$
\frac{\partial u}{\partial x_{1}}(x)=-2\left(x_{1}+a\left(x_{2}, \ldots, x_{n}\right)\right)^{-1} u(x) .
$$

Equation (9) tells us that $u(x)$ is a $C^{\infty}$ function in the variable $x_{1}$. It also enables us to deduce that $u(x)$ is a real-analytic function in $x_{1}$.

Explicitly working out the Taylor expansion of $u(x)$ about one fixed value $x_{1}=x_{1}^{\prime}$ we have

$$
u(x)=-2\left(a\left(x_{2}, \ldots, x_{n}\right)+x_{1}\right)^{-2} b\left(x_{1}^{\prime}, x_{2}, \ldots, x_{n}\right) .
$$

So

$$
w(x)=\left(a\left(x_{2}, \ldots, x_{n}\right)+x_{1}\right)^{-1} b\left(x_{1}^{\prime}, x_{2}, \ldots, x_{n}\right)+c\left(x_{2}, \ldots, x_{n}\right),
$$

where $a, b$ and $c$ are $A_{n}$-valued functions.

We may also easily deduce

Proposition 6. Suppose that $w: V \hookrightarrow \mathbb{R}^{n}$ is a $C^{3}$ diffeomorphism and $\left(\partial^{2} w / \partial x_{1}^{2}\right)(x)=0$ on some neighbourhood of $x_{0} \in V$. Then on that neighbourhood we have

$$
w(x)=x_{1} a^{\prime}\left(x_{2}, \ldots, x_{n}\right)+b^{\prime}\left(x_{2}, \ldots, x_{n}\right),
$$

where $a^{\prime}$ and $b^{\prime}$ are $A_{n}$-valued functions. 
Now using elementary continuity arguments we have, from Propositions 5 and 6 ,

Proposition 7. Suppose that $w: V \hookrightarrow \mathbb{R}^{n}$ is a $C^{3}$ diffeomorphism satisfying $\{S, w\}_{e_{1}}=0$ for all $x \in V$. If $\left(\partial^{2} w / \partial x_{1}^{2}\right)\left(x_{0}\right) \neq 0$ for some $x_{0} \in V$, then $\left(\partial^{2} w / \partial x_{1}^{2}\right)(x) \neq 0$ for any $x \in V$.

We now deduce

Lemma 6. The function $c\left(x_{2}, \ldots, x_{n}\right)$ appearing in $(7)$ is a vector-valued function.

Outline proof. The result follows immediately from allowing the term $x_{1}$, on the right hand side of $(7)$, to vary.

We now see that

$$
w(x)-c\left(x_{2}, \ldots, x_{n}\right)=\left(a\left(x_{2}, \ldots, x_{n}\right)+x_{1}\right)^{-1} b\left(x_{2}, \ldots, x_{n}\right)
$$

is a vector. As we can take the Kelvin inverse of the left hand side of (11), we see that $b\left(x_{2}, \ldots, x_{n}\right)$ is invertible in $A_{n}$. By now allowing $x_{1}$ to vary we have, from (11),

LEMMA 7. $b\left(x_{2}, \ldots, x_{n}\right)^{-1} a\left(x_{2}, \ldots, x_{n}\right)$ is a vector, and so is $b\left(x_{2}, \ldots\right.$ $\left.\ldots, x_{n}\right)$.

As a consequence of Lemma 7 we have

LEMMA 8. The function $a\left(x_{2}, \ldots, x_{n}\right)$ lies in the subspace of $A_{n}$ spanned by the set $\left\{1, e_{i} e_{j}: 1 \leq i<j \leq n\right\}$.

As a consequence of all this we can rewrite (7) as

$$
w(x)=\left(\lambda_{1}\left(x_{2}, \ldots, x_{n}\right)+x_{1} \mu_{1}\left(x_{2}, \ldots, x_{n}\right)\right)^{-1}+\gamma_{1}\left(x_{2}, \ldots, x_{n}\right)
$$

where $\lambda_{1}, \mu_{1}$, and $\gamma_{1}$ are all vectors.

Similar calculations tell us that the functions $a^{\prime}\left(x_{2}, \ldots, x_{n}\right)$ and $b^{\prime}\left(x_{2}, \ldots, x_{n}\right)$ appearing in $(10)$ are vectors.

(10) and (12) give us

THEOREM 1. Suppose that $w: V \hookrightarrow \mathbb{R}^{n}$ is a $C^{3}$ diffeomorphism satisfying $\{S, w\}_{y}=0$ for each $y \in S^{n-1}$. Then for any line $l \subseteq \mathbb{R}^{n}$ with $l \cap V \neq \emptyset$, on each connected line segment of $V \cap l$ the diffeomorphism $w$ is the restriction of a Möbius transformation on $\mathbb{R}^{n} \cup\{\infty\}$.

In fact, elementary geometry and continuity arguments give us

Theorem 2. Suppose that $w: V \hookrightarrow \mathbb{R}^{n}$ is a $C^{3}$ diffeomorphism satisfying $\{S, w\}_{y}=0$ for each $y \in S^{n-1}$. Then for any line $l \subseteq \mathbb{R}^{n}$ with $l \cap V \neq \emptyset$, $\left.w\right|_{V \cap l}$ is the restriction of a Möbius transformation on $\mathbb{R}^{n} \cup\{\infty\}$.

It might initially be suspected that if $w: V \hookrightarrow \mathbb{R}^{n}$ is $C^{3}$ diffeomorphism and $\{S, w\}_{e_{j}}=0$ for $j=1, \ldots, n$ then $w(x)=(a(L x)+b)(c(L x)+d)^{-1}$ 
where $\left(\begin{array}{ll}a & b \\ c & d\end{array}\right)$ is a Vahlen matrix and $L: \mathbb{R}^{n} \rightarrow \mathbb{R}^{n}$ is an isomorphism. Unfortunately, this is not true.

Consider $w\left(x_{1} e_{1}+x_{2} e_{2}\right)=\left(1 / x_{1}\right) e_{1}+\left(1 / x_{2}\right) e_{2}$. Then $\{S, w\}_{e_{1}}=$ $\{S, w\}_{e_{2}}=0$, but $w\left(x_{1} e_{1}+x_{2} e_{2}\right)$ is not a Möbius transformation. Bearing the example in mind we shall continue to look at $C^{3}$ diffeomorphisms whose generalized Schwarzian vanishes at all points in $V$ and in all directions. First we prove:

Proposition 8. Suppose that $w: V \hookrightarrow \mathbb{R}^{n}$ is a $C^{3}$ diffeomorphism and $\{S, w\}_{y}=0$ for $y \in S^{n-1}$. Suppose also that on each line $l$ with $V \cap l \neq \emptyset$ we have

$$
w(x)=\left(\lambda_{l}\left(x_{2}^{\perp}\right)+x_{l} \mu_{l}\left(x_{l}^{\perp}\right)\right)^{-1}+\gamma_{l}\left(x_{l}^{\perp}\right),
$$

where $x_{l}^{\perp}$ is a variable independent of $x_{l}$, and $x_{l}$ is a parametrization of $l$. Then $\gamma_{l}\left(x_{l}^{\perp}\right)$ is a constant.

Proof. Choose a point $x_{0} \in V$, and a ball $B\left(x_{0}, r\right)$. For each ray $r_{x_{0}}$ passing through $x_{0}$ we have

$$
\begin{aligned}
w(x)=\left(\lambda\left(x_{0}\right)\left(\theta_{1}, \ldots, \theta_{n-1}\right)+\left|r_{x_{0}}\right| \mu\left(x_{0}\right)\left(\theta_{1}, \ldots, \theta_{n-1}\right)\right)^{-1} & \\
& +\gamma_{x_{0}}\left(\theta_{1}, \ldots, \theta_{n-1}\right),
\end{aligned}
$$

where $\theta_{1}, \ldots, \theta_{n-1}$ is a parametrization of $S^{n-1}$. So on each ray $w(x)$ has a unique continuation.

From (14) we have $\lim _{\left|r_{x_{0}}\right| \rightarrow \infty} w(x)=\gamma_{x_{0}}\left(\theta_{1}^{\prime}, \ldots, \theta_{n-1}^{\prime}\right)$, where $\left(\theta_{1}^{\prime}, \ldots, \theta_{n-1}^{\prime}\right) \in \gamma_{x_{0}} \cap S^{n-1}$. Similarly, for $x_{1} \in B\left(x_{0}, r\right) \backslash\left\{x_{0}\right\}$ we have

$$
w(x)-\left(\lambda_{x_{1}}\left(\theta_{1}, \ldots, \theta_{n-1}\right)+\left|r_{x_{1}}\right| \mu_{x_{1}}\left(\theta_{1}, \ldots, \theta_{n-1}\right)\right)^{-1}+\gamma_{x_{1}}\left(\theta_{1}, \ldots, \theta_{n-1}\right)
$$

and therefore $\lim _{\left|r_{x_{1}}\right| \rightarrow \infty} w(x)=\gamma_{x_{1}}\left(\theta_{1}^{\prime}, \ldots, \theta_{n-1}^{\prime}\right)$.

Now choose a continuous function $z:(0, \infty) \rightarrow \mathbb{R}^{n}$ so that $z(0)=x_{0}$ and $z(t)$ is asymptotic to the ray $r_{x_{1}}$. As $\lambda_{l}, \mu_{l}$ and $\gamma_{l}$ are continuous we obtain $\lim _{t \rightarrow \infty} w(z(t))=\gamma_{x_{0}}\left(\theta_{1}^{\prime}, \ldots, \theta_{n-1}^{\prime}\right)$. Consequently, $\gamma_{x_{1}}\left(\theta_{1}^{\prime}, \ldots, \theta_{1}^{\prime}\right)=$ $\gamma_{x_{0}}\left(\theta_{1}^{\prime}, \ldots, \theta_{n-1}^{\prime}\right)$. As this is true for each $x_{1} \in B\left(x_{0}, r\right), \gamma_{l}\left(x_{l}^{\perp}\right)$ is a constant.

We shall denote this constant vector by $\gamma$. Trivially we have:

Lemma 9. Suppose that $w(x)$ is as in Proposition 8. Then the $C^{3}$ diffeomorphism $w(x)-\gamma$ also has the generalized Schwarzian zero for all $y \in S^{n-1}$. Moreover, on each line $l$ we have

$$
w(x)-\gamma=\left(\lambda_{l}\left(x_{l}^{\perp}\right)+x_{l} \mu_{l}\left(x_{l}^{\perp}\right)\right)^{-1} .
$$

Via direct computation we may deduce

Proposition 9. Suppose that $w: V \hookrightarrow \mathbb{R}^{n}$ is a $C^{3}$ diffeomorphism and $\{S, w(x)\}_{y}=0$ for all $x \in V$ and all $y \in S^{n-1}$. Then $\left\{S, w(x)^{-1}\right\}_{y}=0$ for all $x \in V$ and all $y \in S$. 
On taking the Kelvin inverse of $w(x)-\gamma$ it follows from Proposition 6 that on any two-dimensional hyperspace of $\mathbb{R}^{n}$ spanned by $e_{i}$ and $e_{j}$ and intersecting $V$ we have

$$
\begin{aligned}
(w(x)-\gamma)^{-1}= & v_{1}\left(x_{1}, \ldots, \widehat{x}_{i}, \ldots, \widehat{x}_{j}, \ldots, x_{n}\right) \\
& +x_{i} v_{i}\left(x_{1}, \ldots, \widehat{x}_{i}, \ldots, \widehat{x}_{j}, \ldots, x_{n}\right) \\
& +x_{j} v_{j}\left(x_{1}, \ldots, \widehat{x}_{i}, \ldots, \widehat{x}_{j}, \ldots, x_{n}\right) \\
& +x_{i} x_{j} v_{i j}\left(x_{1}, \ldots, \widehat{x}_{i}, \ldots, \widehat{x}_{j}, \ldots, x_{n}\right),
\end{aligned}
$$

where $v_{1}, v_{i}, v_{j}$ and $v_{i j}$ are vectors. On setting $x_{i}=u_{i}-u_{j}$ and $x_{j}=u_{i}+u_{j}$ it now follows from Propositions 6 and 9 that $v_{i j}=0$. Consequently, we have

Theorem 3. Suppose that $w: V \hookrightarrow \mathbb{R}^{n}$ is a $C^{3}$ diffeomorphism satisfying $\{S, w\}_{y}=0$ for each $y \in S^{n-1}$. Then there is an isomorphism $L: \mathbb{R}^{n} \rightarrow \mathbb{R}^{n}$ and a Vahlen matrix $\left(\begin{array}{ll}a & b \\ c & d\end{array}\right)$ such that $w(x)=(a(L x)+b)(c(L x)+d)^{-1}$.

We now turn to look at other properties of this generalized Schwarzian. We begin with

TheOREm 4. Suppose that $w: V \hookrightarrow \mathbb{R}^{n}$ is a $C^{3}$ diffeomorphism, and $\left(\begin{array}{ll}a & b \\ c & d\end{array}\right) \in V(n) \backslash \mathbb{R}_{+}^{n}$. Then

$$
\left\{S,(a w+b)(c w+d)^{-1}\right\}_{y}=(w \widetilde{c}+\widetilde{d})^{-1}\{S, w\}_{y}(w \widetilde{c}+\widetilde{d}) .
$$

Outline proof. When $c=0$, the result follows from (4). When $c \neq 0$ we have $(a w+b)(c w+d)^{-1}=a c^{-1}+\lambda(c w \widetilde{c}+d \widetilde{c})^{-1}$ where $\lambda \neq 1$. The result now follows from Proposition 3.

As $c w \widetilde{c}+d \widetilde{c}$ is a vector in $\mathbb{R}^{n}, c w+d$ can be expressed as a product of vectors in $\mathbb{R}^{n}$. Consequently, (15) can be rewritten as

$$
\left\{S,(a w+b)(c w+d)^{-1}\right\}_{y}=\operatorname{sgn}(c w+d) \frac{(c w+d)\{S, w\}_{y}(c \widetilde{w}+d)}{|c w+d|^{2}}
$$

where $\operatorname{sgn}(c w+d)$ is the sign of $(c w+d)(c \widetilde{w}+d)$.

If we dictate that the basis (1) is an orthonormal basis for $A_{n}$ then (16) yields

Proposition 10. If $w: V \hookrightarrow \mathbb{R}^{n}$ is a $C^{3}$ diffeomorphism and $\left(\begin{array}{ll}a & b \\ c & d\end{array}\right) \in$ $V(n) \backslash \mathbb{R}^{+}$then for each $y_{1}, y_{2} \in S^{n-1}$ we have

$$
\begin{aligned}
\left\langle\{S, w\}_{y_{1}},\{S, w\}_{y_{2}}\right\rangle & \\
= & \left\langle\left\{S,(a w+b)(c w+d)^{-1}\right\}_{y_{1}},\left\{S,(a w+b)(c w+d)^{-1}\right\}_{y_{2}}\right\rangle .
\end{aligned}
$$

If $w: V \hookrightarrow \mathbb{R}^{n}$ is a $C^{3}$ diffeomorphism we shall let $\{S, w\}_{y, 0}$ denote the identity component of $\{S, w\}_{y}$, while $\{S, w\}_{y, i j}$ denotes the bivector component of $\{S, w\}_{y}$, that is, the component spanned by $\left\{e_{i} e_{j}: 1 \leq i<j \leq n\right\}$. 
Moreover, $\{S, w\}_{y, i j k l}$ denotes the four-vector component of $\{S, w\}_{y}$, spanned by $\left\{e_{i} e_{j} e_{k} e_{l}: 1 \leq i<j<k<l \leq n\right\}$. As

$$
(c w+d) e_{i} e_{j}(c \widetilde{w}+d)=\frac{(c w+d) e_{i}(c \widetilde{w}+d)(c w+d) e_{j}(c \widetilde{w}+d)}{(c w+d)(c \widetilde{w}+d)},
$$

we have from (16)

Proposition 11. Suppose $w: V \hookrightarrow \mathbb{R}^{n}$ is a $C^{3}$ diffeomorphism and $\left(\begin{array}{ll}a & b \\ c & d\end{array}\right) \in V(n) \backslash \mathbb{R}^{+}$. Then

$$
\begin{aligned}
& \left\{S,(a w+b)(c w+d)^{-1}\right\}_{y, i j}=\operatorname{sgn}(c w+d) \frac{(c w+d)\{S, w\}_{y, i j}(c \widetilde{w}+d)}{|c w+d|^{2}} \\
& \left\{S,(a w+b)(c w+d)^{-1}\right\}_{y, i j k l} \\
& \quad=\operatorname{sgn}(c w+d) \frac{(c w+d)\{S, w\}_{y, i j k l}(c \widetilde{w}+d)}{|c w+d|^{2}}
\end{aligned}
$$

We also have

Proposition 12. Suppose $w: V \hookrightarrow \mathbb{R}^{n}$ is a $C^{3}$ diffeomorphism and $\left(\begin{array}{ll}a & b \\ c & d\end{array}\right) \in V(n) \backslash \mathbb{R}^{+}$. Then

$$
\left\{S,(a w+b)(c w+d)^{-1}\right\}_{y, 0}=\{S, w\}_{y, 0} .
$$

Propositions 11 and 12 give us

$$
\begin{aligned}
\left\langle\left\{S,(a w+b)(c w+d)^{-1}\right\}_{y_{1}, i j},\{S,(a w+b)\right. & \left.\left.(c w+d)^{-1}\right\}_{y_{2}, i j}\right\rangle \\
& =\left\langle\{S, w\}_{y_{1}, i j},\{S, w\}_{y_{2}, i j}\right\rangle
\end{aligned}
$$

and

$$
\begin{array}{r}
\left\langle\left\{S,(a w+b)(c w+d)^{-1}\right\}_{y_{1}, i j k l},\left\{S,(a w+b)(c w+d)^{-1}\right\}_{y_{2}, i j k l}\right\rangle \\
=\left\langle\{S, w\}_{y_{1}, i j k l},\{S, w\}_{y_{2}, i j k l}\right\rangle .
\end{array}
$$

Explicitly computing $\{S, w\}_{y, 0}$ we get

$$
\left\langle w_{y y y}, w_{y}\right\rangle\left|w_{y}\right|^{-2}-\frac{3}{2}\left\langle w_{y y}, w_{y}\right\rangle^{2}\left|w_{y}\right|^{-4}+\frac{3}{2}\left|w_{y y}\right|^{2}\left|w_{y}\right|^{-2} .
$$

This expression corresponds to one of the generalizations of the Schwarzian derivative given in [3].

Using differential forms we find that $\{S, w\}_{y, i j}$ is equivalent to

$$
w_{y} \wedge w_{y y y}-3\left\langle w_{y}, w_{y y}\right\rangle\left(w_{y} \wedge w_{y y}\right)\left|w_{y}\right|^{-4}
$$

where $w_{y}, w_{y y y}$ are all regarded as 1 -forms. This expression is identical to the second generalized Schwarzian derivative appearing in [3].

We now show that the usual method of obtaining a chain rule for the Schwarzian in one complex variable breaks down.

Suppose now $g(w): V \hookrightarrow \mathbb{R}^{n}$ is a $C^{3}$ diffeomorphism. Ideally we would like to obtain an expression for $\{S, g(w)\}_{y}$ in terms of $\{S, g\}_{w_{y}}$ and $\{S, w\}_{y}$. First we note that $g(w)_{y y y}$ contains the term $D g_{w(x)} w_{y y y}$, while $g(w)_{y y}$ 
contains the term $D g_{w(x)} w_{y y}$, and $g(w)_{y}$ is equal to $D g_{w(x)} w_{y}$. We could re-express $D g_{w(x)} w_{y y y}, D g_{w(x)} w_{y y}$ and $D g_{w(x)} w_{y}$ as $a_{1}(x, y) w_{y y y} \widetilde{a}_{1}(x, y)$, $a_{2}(x, y) w_{y y} \widetilde{a}_{2}(x, y)$ and $a_{3}(x, y) w_{y} \widetilde{a}_{3}(x, y)$, respectively, where $a_{j}(x, y)=$ $b_{j, 1}(x, y) \ldots b_{j, n_{j}}(x, y)$ with $b_{i, j}(x, y) \in \mathbb{R}^{n} \backslash\{0\}$ for $j=1,2,3$ and $1 \leq i \leq n_{j}$.

In general $a_{j}(x, y)=a_{k}(x, y)$ only for $j=k$ so we are unable to use this approach to extend the chain rule given in Theorem 4 to obtain a generalization of the Schwarzian chain rule described in [10].

3. Besides $A_{n}$ we can also construct [14] the Clifford algebra $A_{p, q}$ from the vector space $\mathbb{R}^{p, q}$. The space $\mathbb{R}^{p, q}$ is spanned by the elements $f_{1}, \ldots, f_{p}$, $e_{p+1}, \ldots, e_{p+q}$, and it is endowed with the quadratic form $\langle$,$\rangle , where$

$$
\langle x, x\rangle=x_{1}^{2}+\ldots+x_{p}^{2}-x_{p+1}^{2}-\ldots-x_{p+q}^{2}
$$

for $x=x_{1} f_{1}+\ldots+x_{p} f_{p}+x_{p+1} e_{p+1}+\ldots+x_{p+q} e_{p+q}$. To construct $A_{p, q}$ we define the relations

$$
e_{i} f_{j}=-f_{j} e_{i}, \quad e_{i} e_{j}+e_{j} e_{i}=-2 \delta_{i j}, \quad f_{i} f_{j}+f_{j} f_{i}=2 \delta_{i j} .
$$

It may now be deduced that $A_{p, q}$ has dimension $2^{p+q}$. When $p=0$ and $q=n$ we have $A_{0, n}=A_{n}$. It is straightforward to extend the antiautomorphisms $\sim$ and - to $A_{p, q}$ (see [14]). Also, we have the following extension of the Pin group:

$$
\begin{aligned}
\operatorname{Pin}(p, q)=\left\{a \in A_{p, q}: a=a_{1} \ldots a_{k},\right. & k \in \mathbb{N} \text { and } a_{j} \in \mathbb{R}^{p, q} \\
& \text { where } \left.a_{j}^{2}= \pm 1 \text { for } 1 \leq j \leq k\right\} .
\end{aligned}
$$

Moreover [14], $\langle a x \widetilde{a}, a x \widetilde{a}\rangle=\langle x, x\rangle$ for each $a \in \operatorname{Pin}(p, q)$. It may easily be verified that $\operatorname{Pin}(p, q)$ is a covering group of

$$
O(p, q)=\left\{T: \mathbb{R}^{p, q} \rightarrow \mathbb{R}^{p, q}:\right.
$$

$T$ is linear and $\langle T x, T x\rangle=\langle x, x\rangle$ for all $\left.x \in \mathbb{R}^{p, q}\right\}$.

If we take the closure, within the algebra $A_{p, q}(2)$ (of $2 \times 2$ matrices with coefficients in $A_{p, q}$ ), of the group generated by

$$
\begin{aligned}
& \left\{\left(\begin{array}{cc}
a & 0 \\
0 & \widetilde{a}^{-1}
\end{array}\right),\left(\begin{array}{ll}
1 & v \\
0 & 1
\end{array}\right),\left(\begin{array}{cc}
0 & \pm 1 \\
1 & 0
\end{array}\right),\left(\begin{array}{cc}
\lambda & 0 \\
0 & \lambda^{-1}
\end{array}\right):\right. \\
& \left.a \in \operatorname{Pin}(p, q), v \in \mathbb{R}^{p, q}, \lambda \in \mathbb{R}^{+}\right\}
\end{aligned}
$$

we obtain a new group which we denote by $V(p, q)$. Again, when $p=0$ and $q=n$ we obtain $V(n) \backslash \mathbb{R}^{+}$.

We could also take the closure, within $A_{p, q}(2)$, of the group generated 
by

$$
\begin{gathered}
\left\{\left(\begin{array}{cc}
a & 0 \\
0 & \widetilde{a}^{-1}
\end{array}\right),\left(\begin{array}{cc}
1 & v \\
0 & 1
\end{array}\right),\left(\begin{array}{cc}
0 & \pm 1 \\
1 & 0
\end{array}\right),\left(\begin{array}{cc}
\lambda & 0 \\
0 & \lambda^{-1}
\end{array}\right): a=a_{1} \ldots a_{r}, r \in \mathbb{N},\right. \\
\left.a_{j} \in \mathbb{R}+\mathbb{R}^{p, q} \text { with } a_{j}^{2}= \pm 1 \text { for } 1 \leq j \leq r, v \in \mathbb{R}+\mathbb{R}^{p, q}, \lambda \in \mathbb{R}^{+}\right\}
\end{gathered}
$$

where $\mathbb{R}+\mathbb{R}^{p, q}$ is spanned by $1, f_{1}, \ldots, f_{p}, e_{p+1}, \ldots, e_{p+q}$. We denote this group by $V_{0}(p, q)$. When $p=0$ and $q=n$ we have $V_{0}(p, q)=V_{0}(n) / \mathbb{R}^{+}$.

For $x=x_{0}+x_{1} f_{1}+\ldots+x_{p} f_{p} \in \mathbb{R}+\mathbb{R}^{p, 0}$ we have $x \bar{x}=x_{0}^{2}-x_{1}^{2}-\ldots-x_{p}^{2}$, so $\mathbb{R}+\mathbb{R}^{3,0}$ inherits the same structure as the four-dimensional Minkowski space. On making the identifications

$$
\begin{aligned}
1 \mapsto\left(\begin{array}{ll}
1 & 0 \\
0 & 1
\end{array}\right), & f_{1} \mapsto\left(\begin{array}{cc}
1 & 0 \\
0 & -1
\end{array}\right), \\
f_{2} \mapsto\left(\begin{array}{ll}
0 & 1 \\
1 & 0
\end{array}\right), & f_{3} \mapsto\left(\begin{array}{cc}
0 & i \\
-i & 0
\end{array}\right)
\end{aligned}
$$

we see [8] that $\mathbb{R}+\mathbb{R}^{3,0}$ is identified with $H_{2}$, the space of $2 \times 2$ Hermitean matrices. Also, for

$$
A=\left(\begin{array}{cc}
x_{0}+x_{1} & x_{2}+i x_{3} \\
x_{2}-i x_{3} & x_{0}-x_{1}
\end{array}\right) \in H_{2}
$$

we have $\operatorname{det} A=x_{0}^{2}-x_{1}^{2}-x_{2}^{2}-x_{3}^{2}$. Using the identifications (17) it is straightforward calculation to see that $A_{3,0}$ is isomorphic to $\mathbb{C}(2)$, the algebra of $2 \times 2$ complex matrices.

Via this isomorphism it may now be deduced from the description of $V_{0}(p, q)$ that

$$
\begin{aligned}
V_{0}(3,0) \cong U(2,2) & =\left\{\left(\begin{array}{ll}
A & B \\
C & D
\end{array}\right): A, B, C, D \in \mathbb{C}(2)\right. \text { and } \\
& \left.\left(\begin{array}{ll}
A & B \\
C & D
\end{array}\right)\left(\begin{array}{cc}
0 & I_{2} \\
-I_{2} & 0
\end{array}\right)\left(\begin{array}{ll}
\bar{A}^{T} & \bar{C}^{T} \\
\bar{B}^{T} & \bar{D}^{T}
\end{array}\right)= \pm\left(\begin{array}{cc}
0 & I_{2} \\
-I_{2} & 0
\end{array}\right)\right\},
\end{aligned}
$$

where $I_{2}=\left(\begin{array}{ll}1 & 0 \\ 0 & 1\end{array}\right)$.

In greater generality, we have the group

$$
\begin{aligned}
U(n, n)= & \left\{\left(\begin{array}{ll}
A & B \\
C & D
\end{array}\right): A, B, C, D \in \mathbb{C}(n)\right. \text { and } \\
& \left.\left(\begin{array}{ll}
A & B \\
C & D
\end{array}\right)\left(\begin{array}{cc}
0 & I_{n} \\
-I_{n} & 0
\end{array}\right)\left(\begin{array}{ll}
\bar{A}^{T} & \bar{C}^{T} \\
\bar{B}^{T} & \bar{D}^{T}
\end{array}\right)= \pm\left(\begin{array}{cc}
0 & I_{n} \\
-I_{n} & 0
\end{array}\right)\right\},
\end{aligned}
$$

where $I_{n}$ is the $n \times n$ identity matrix.

We shall let $H_{n}$ denote the space of $n \times n$ Hermitean matrices. 
As $U(n, n)$ is the closure of the subgroup of $\mathbb{C}(2 n)$ generated by the set (18) $\left\{\left(\begin{array}{cc}A & 0 \\ 0 & \left(\bar{A}^{T}\right)^{-1}\end{array}\right),\left(\begin{array}{cc}I_{n} & B \\ 0 & I_{n}\end{array}\right),\left(\begin{array}{cc}0 & \pm I_{n} \\ I_{n} & 0\end{array}\right): A \in \mathbb{C}(n), B \in H(n)\right\}$ we can deduce that for each $\left(\begin{array}{ll}A & B \\ C & D\end{array}\right) \in U(n, n)$ the function

$$
\operatorname{det}_{C, D}: H_{n} \rightarrow \mathbb{C}, \quad X \mapsto \operatorname{det}(C X+D)
$$

is non-zero on an open, dense subset of $H_{n}$. Hence $(A X+B)(C X+D)^{-1}$ is well defined on this open, dense set. Moreover, using (18) we see that $(A X+B)(C X+D)^{-1} \in H_{n}$ whenever $(C X+D)^{-1}$ is defined.

The fractional linear transformation $(A X+B)(C X+D)^{-1}$ has previously been described in $[7,9]$, and elsewhere.

4. From the previous section we may deduce:

Proposition 13. Suppose that $\left(\begin{array}{ll}A & B \\ C & D\end{array}\right) \in U(n, n)$, and $z \in H_{n} \backslash\{0\}$. Let $\Phi(X)=(A X+B)(C X+D)^{-1}$. Then

$$
\Phi(X)_{z z z} \Phi(X)_{z}^{-1}-\frac{3}{2}\left\{\Phi(X)_{z z} \Phi(X)_{z}^{-1}\right\}^{2}=0,
$$

where $\Phi(X)_{z}$ denotes the partial derivative of $\Phi(X)$ in the direction of $z$.

In particular, Proposition 13 tells us that the group $U(2,2)$, used to describe Möbius transformations in Minkowski space, has a generalized Schwarzian derivative associated with it.

Proposition 13 leads us to the following definition.

Definition 5. Suppose that $V$ is a domain in $H_{n}$ and $h: V \hookrightarrow H_{n}$ is a $C^{3}$ diffeomorphism, and for some direction $z \in H \backslash\{0\}$ the element $h(X)_{z}$ is invertible. Then

$$
h(X)_{z z z} h(X)_{z}^{-1}-\frac{3}{2}\left\{h(X)_{z z} h(X)_{z}^{-1}\right\}^{2}
$$

is called the $U(n, n)$ Schwarzian derivative of $h(X)$ in the direction of $z$. We denote it by

$$
\left\{S_{U(n, n)}, h(X)\right\}_{z}
$$

By similar arguments to those used to deduce Theorem 4 we have

TheOrem 5. Suppose that $\left(\begin{array}{ll}A & B \\ C & D\end{array}\right) \in U(n, n), V$ is a domain in $H_{n}$ and $h: V \hookrightarrow H_{n}$ is a $C^{3}$ diffeomorphism. Suppose that for some direction $z \in H_{n} \backslash\{0\}$ the element $h(X)_{z}$ is invertible. Then

$$
\begin{aligned}
&\left\{S_{U(n, n)},(\right.\left.A h(X)+B)(h(X)+D)^{-1}\right\} \\
&=\left(h(X) \bar{C}^{T}+D^{T}\right)^{-1}\left\{S_{U(n, n)}, h(X)\right\}_{z}\left(h(X) \bar{C}^{T}+\bar{D}^{T}\right) .
\end{aligned}
$$


5. Besides the groups $V(n)$ and $U(n, n)$ we can also associate a Schwarzian with the real symplectic group

$$
\begin{aligned}
\operatorname{Sp}(n, \mathbb{R})= & \left\{\left(\begin{array}{ll}
A & B \\
C & D
\end{array}\right): A, B, C, D \in \mathbb{R}(n)\right. \text { and } \\
& \left.\left(\begin{array}{ll}
A & B \\
C & D
\end{array}\right)\left(\begin{array}{cc}
0 & I_{n} \\
-I_{n} & 0
\end{array}\right)\left(\begin{array}{ll}
A^{T} & C^{T} \\
B^{T} & D^{T}
\end{array}\right)=\left(\begin{array}{cc}
0 & I_{n} \\
-I_{n} & 0
\end{array}\right)\right\},
\end{aligned}
$$

described in $[7,9]$, and elsewhere. $\operatorname{Sp}(n, \mathbb{R})$ can be seen as the closure of the subgroup of $\mathbb{R}(2 n)$ with generators the set

$$
\left\{\left(\begin{array}{cc}
A & 0 \\
0 & \left(A^{T}\right)^{-1}
\end{array}\right),\left(\begin{array}{cc}
1 & B \\
0 & 1
\end{array}\right),\left(\begin{array}{cc}
0 & -1 \\
1 & 0
\end{array}\right): A, B \in \mathbb{R}(n)\right\} .
$$

By similar arguments to those used in Section 3 we find that for $\left(\begin{array}{ll}A & B \\ C & D\end{array}\right) \in$ $\operatorname{Sp}(n, \mathbb{R})$ the matrix $C X+D$ is invertible on an open, dense subset of $S_{n}=$ $\left\{X \in \mathbb{R}(n): X^{T}=X\right\}$. Moreover, $(A X+B)(C X+D)^{-1} \in S_{n}$ on this set.

Definition 6. Suppose that $V$ is a domain in $S_{n}$ and $h: V \hookrightarrow S_{n}$ is a $C^{3}$ diffeomorphism. Suppose also for some direction $z \in S_{n} \backslash\{0\}$ the element $h(X)_{z}$ is invertible. Then

$$
h(X)_{z z z} h(X)_{z}-\frac{3}{2}\left\{h(X)_{z z} h(X)_{z}^{-1}\right\}^{2}
$$

is called the $\operatorname{Sp}(n, \mathbb{R})$ Schwarzian derivative of $h(X)$ in the direction of $z$. We denote it by $\left\{S_{\mathrm{Sp}(n, \mathbb{R})}, h(X)\right\}_{z}$.

Theorem 6. Suppose that $\left(\begin{array}{ll}A & B \\ C & D\end{array}\right) \in \operatorname{Sp}(n, \mathbb{R})$. Then

$$
\begin{aligned}
\left\{S_{\mathrm{Sp}(n, \mathbb{R})},\right. & \left.(A h(X)+B)(C h(X)+D)^{-1}\right\}_{z} \\
= & \left(h(X) C^{T}+D^{T}\right)^{-1}\left\{S_{\mathrm{Sp}(n, \mathbb{R})}, h(X)\right\}_{z}\left(h(X) C^{T}+D^{T}\right) .
\end{aligned}
$$

If $h(X)=X$ for all $X \in S_{n}$ then

$$
\left\{S_{\mathrm{Sp}(n, \mathbb{R})},(A X+B)(C X+D)^{-1}\right\}_{z}=0 .
$$

By similar arguments we may introduce a Schwarzian derivative and an analogue of Theorems 5 and 6 for the quaternionic group

$$
\begin{aligned}
H(n, n)= & \left\{\left(\begin{array}{ll}
A & B \\
C & D
\end{array}\right) \in \mathbb{H}(2 n):\right. \\
& \left.\left(\begin{array}{cc}
A & B \\
C & D
\end{array}\right)\left(\begin{array}{cc}
0 & I_{n} \\
-I_{n} & 0
\end{array}\right)\left(\begin{array}{ll}
\bar{A}^{T} & \bar{C}^{T} \\
\bar{B}^{T} & \bar{D}^{T}
\end{array}\right)=\left(\begin{array}{cc}
0 & I_{n} \\
-I_{n} & 0
\end{array}\right)\right\},
\end{aligned}
$$

where - here denotes quaternionic conjugation.

6. In this final section we briefly describe how the results of the previous two sections carry through to the group $V(p, q)$.

First suppose that $\left(\begin{array}{ll}a & b \\ c & d\end{array}\right) \in V(p, q)$. Then it follows from the description of $V(p, q)$ given in Section 3 that $(c x+d)(\widetilde{x}+d)$ is real-valued, non-zero 
on an open dense subset of $\mathbb{R}^{p, q}$. Consequently, $(a x+b)(c x+d)^{-1}$ is well defined on this set. Moreover, it follows from our characterization of $V(p, q)$ that $(a x+b)(c x+d)^{-1}$ is a Möbius transformation on $\mathbb{R}^{p, q}$. It is now straightforward to construct a Schwarzian derivative on $\mathbb{R}^{p, q}$ and to obtain an analogue of Theorems 5 and 6 in this setting.

\section{References}

[1] L. V. Ahlfors, Clifford numbers and Möbius transformations in $\mathbb{R}^{n}$, in: Clifford Algebras and their Applications in Mathematical Phisics, J. S. R. Chrisholm and A. K. Common (eds.), NATO Adv. Study Inst. Ser., Ser. C: Math. Phys. Sci., Vol. 183, Reidel, 1986, 167-175.

[2] -, Möbius transformations in $\mathbb{R}^{n}$ expressed through $2 \times 2$ matrices of Clifford numbers, Complex Variables 5 (1986), 215-224.

[3] -, Cross-ratios and Schwarzian derivatives in $\mathbb{R}^{n}$, preprint.

[4] M. F. Atiyah, R. Bott and A. Shapiro, Clifford modules, Topology 3 (1964), 3-38.

[5] K. Carne, The Schwarzian derivative for conformal maps, to appear.

[6] J. Elstrodt, F. Grunewald and J. Mennicke, Vahlen's group of Clifford matrices and Spin-groups, Math. Z. 196 (1987), 369-390.

[7] K. Gross and R. Kunze, Bessel functions and representation theory, II. Holomorphic discrete series and metaplectic representations, J. Funct. Anal. 25 (1977), $1-49$.

[8] H. P. Jakobsen, Intertwining differential operators for $\mathrm{Mp}(n, \mathbb{R})$ and $S U(n, n)$, Trans. Amer. Math. Soc. 246 (1978), 311-337.

[9] H. P. Jakobsen and M. Vergne, Wave and Dirac operators and representations of the conformal group, J. Funct. Anal. 24 (1977), 52-106.

[10] O. Lehto, Univalent Functions and Teichmüller Spaces, Graduate Texts in Math. 109, Springer, 1986.

[11] H. Maass, Automorphe Funktionen von mehreren Veränderlichen und Dirichletsche Reihen, Abh. Math. Sem. Univ. Hamburg 16 (1949), 72-100.

[12] P. Osgood and D. Stowe, The Schwarzian derivative and conformal mapping of Riemannian manifolds, to appear.

[13] -, -, A generalization of Nehari's univalence criterion, to appear.

[14] I. R. Porteous, Topological Geometry, Cambridge Univ. Press, 1981.

[15] K. Th. Vahlen, Ueber Bewegungen und complexe Zahlen, Math. Ann. 55 (1902), 585-593.

Current address:

DEPARTMENT OF PURE MATHEMATICS UNIVERSITY OF SYDNEY SYDNEY, NEW SOUTH WALES 2006 AUSTRALIA
DEPARTMENT OF MATHEMATICAL SCIENCES UNIVERSITY OF ARKANSAS FAYETTEVILLE, ARKANSAS 72701 U.S.A. 